\title{
A SOLAR PLAGE MODEL
}

\author{
A. KUČERA \\ Astronomical Institute of the Slovak Academy of Sciences, \\ CS-05960 Tatranská Lomnica, Czechoslovakia \\ and \\ E. A. BARANOVSKY \\ Crimean Astrophysical Observatory, 334413 Crimea, Ukraine
}

\begin{abstract}
An investigation of a solar plage based on observations of UV, visible, and IR spectral lines is presented. Using model calculations, we have established distributions of the main physical parameters (temperature, density, turbulent velocity) in both plage and quiet-Sun atmospheres. Details of the models are presented and the role of magnetic pressure is discussed.
\end{abstract}

Key words: infrared: stars - Sun: chromosphere - Sun: faculae, plages

\section{Introduction}

The problems of the heating of the solar atmosphere and of solar activity still remain of central interest in solar physics. Previous investigations of the physical properties of solar plages were carried out by many of authors (e.g., Shine and Linsky (1972, 1974), Ayres et al. (1986) and LaBonte (1986a,b). Observations in the far UV spectral region and their interpretations [for example, Kelch and Linsky (1978), Baranovsky and Severny (1979), Basri et al. (1979), Lemaire et al. (1981)] have shown that physical conditions are complicated inside the solar plages and many physical influences - like magnetic fields, velocity fields, a mechanical energy fluxes and radiative losses - play important roles in both the energy and pressure balance of the plage atmosphere. The parameters of the 'average' plage have been obtained through the investigation of many plages. These studies have shown that a credible approach requires complex calculations of line formation. This work describes a further attempt to observe and model solar plages using continua and lines (including the Ca II IR triplet) that are formed over a range of height in the model atmosphere.

\section{Observations and data reduction}

Low-spatial resolution spectral observations have been made using the Horizontal Solar Telescope with Spectrograph (HSTS) at Stara Lesna Observatory. The main parameters of the HSTS were presented in Kučera et al. (1990). A set of nine wellcalibrated photographic line profiles of $\mathrm{H} \alpha, \mathrm{H} \beta, \mathrm{H} \gamma, \mathrm{Ca}$ II $\mathrm{H}$ and $\mathrm{K}$ and the IR triplet (at $849.8 \mathrm{~nm}, 854.2 \mathrm{~nm}$ and $866.2 \mathrm{~nm}$ ), and $\mathrm{HeD}_{3}$ were obtained in both active and quiet regions. The observation lasted 14 minutes (without the calibration measurements); the slit height was the equivalent of $237^{\prime \prime} 5$ and its width 0.3 . All line profiles have been normalized to their nearby continua. We followed the method of Jebsen and Mitchel (1978) to estimate the continua in the UV region for Ca II $\mathrm{H}$ and $\mathrm{K}$ lines. 

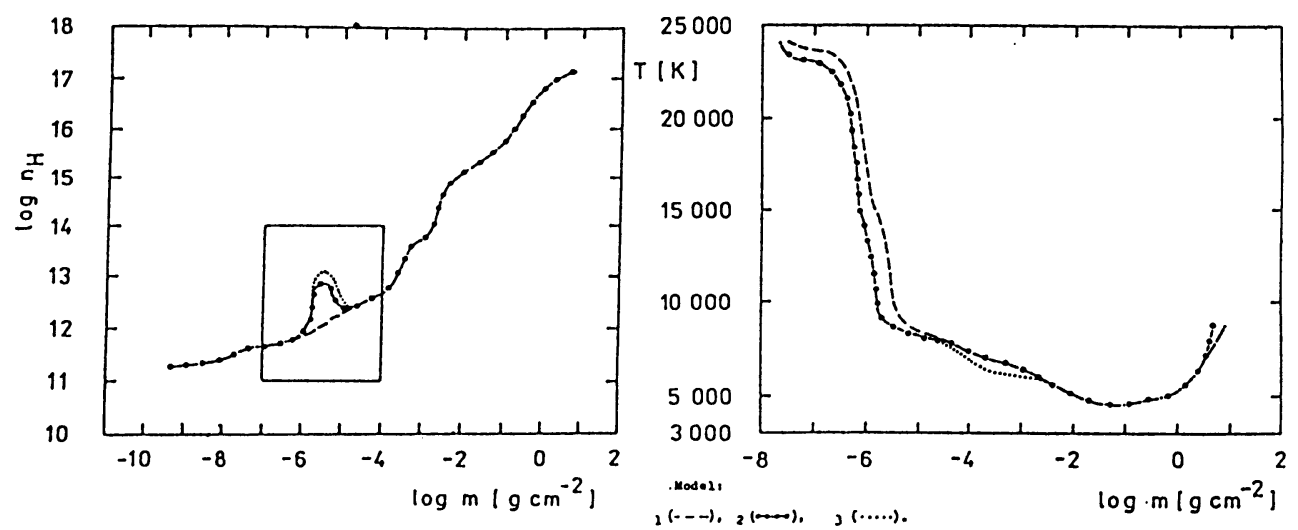

Fig. 1. Hydrogen number density and temperature as a function of mass column densities.

\section{Modelling}

We used a non-LTE computational program based on the Equivalent Two Level Atom (ETLA) approach. The following assumptions were adopted throughout our calculations:

1. A plane-parallel atmosphere without illumination by coronal radiation.

2. Complete redistribution (CRD) in the line transfer calculations.

3. The atmospheric parameters depend on height only.

4. Atomic models consist of 9 levels and continuum for hydrogen, of 6 levels and continuum for $\mathrm{Ca}$ II ion, and of 13 levels and continuum for Helium.

The computational method used is outlined by Baranovsky and Stepanian (1976, 1979), Baranovsky and Severny (1979), and Kučera et al. (1990) - it follows the approach of Avrett and Loeser (1969).

\section{Results and Discussion}

Three models of plage atmosphere were finally selected based on the agreement obtained between the computed profiles and the observations, which fit the observational profiles well. The models differ from each other in the hydrogen number density in the higher layers of the chromosphere as well as in the depth dependence of the temperature. The models are illustrated in Figure 1. Computed and observed profiles of $\mathrm{Ca}$ II K, Ca II IR $866.2 \mathrm{~nm}$ and $\mathrm{He}_{3}$ are shown in Figure 2 with corresponding values of the macroturbulent velocity. (For $\mathrm{He} \mathrm{D}_{3}$ the differences between plage and quiet Sun are shown.) 
TABLE I

Plage Model No. 3

\begin{tabular}{|c|c|c|c|c|c|c|c|}
\hline $\mathrm{N}$ & $\begin{array}{c}\mathrm{h} \\
(\mathrm{km})\end{array}$ & $\begin{array}{c}\mathrm{m} \\
\left(\mathrm{g} \mathrm{cm}^{-2}\right)\end{array}$ & $\tau_{L \alpha}$ & $\begin{array}{c}\mathrm{T} \\
(\mathrm{K})\end{array}$ & $\begin{array}{c}\mathrm{v} \\
\left(\mathrm{km} \mathrm{s}^{-1}\right)\end{array}$ & $\begin{array}{c}n_{H} \\
\left(\mathrm{~cm}^{-3}\right)\end{array}$ & $\begin{array}{c}n_{e} \\
\left(\mathrm{~cm}^{-3}\right)\end{array}$ \\
\hline 1 & 1211.3 & $1.670-24$ & $2.496-3$ & 40850 & 7.0 & $1.900+11$ & $1.872+11$ \\
\hline 2 & 1211.3 & $5.327-10$ & 4.996-3 & 39850 & 7.0 & $1.900+11$ & $1.865+11$ \\
\hline 3 & 1211.3 & $1.456-9$ & $1.000-2$ & 38950 & 7.0 & $2.010+11$ & $1.972+11$ \\
\hline 4 & 1211.2 & 2.973-9 & $2.001-2$ & 37450 & 7.0 & $2.250+11$ & $2.212+11$ \\
\hline 5 & 1211.2 & $5.210-9$ & $4.006-2$ & 35450 & 7.0 & $2.380+11$ & $2.320+11$ \\
\hline 6 & 1211.1 & 8.534-9 & $8.018-2$ & 34040 & 7.0 & $2.650+11$ & $2.604+11$ \\
\hline 7 & 1211.0 & $1.319-8$ & $1.605-1$ & 31930 & 7.0 & $2.720+11$ & $2.648+11$ \\
\hline 8 & 1210.9 & $1.837-8$ & $3.212-1$ & 29120 & 7.0 & $3.050+11$ & $2.996+11$ \\
\hline 9 & 1210.8 & $2.388-8$ & $6.428-1$ & 26610 & 7.0 & $3.440+11$ & $3.375+11$ \\
\hline 10 & 1210.7 & $2.973-8$ & 1.287 & 24400 & 7.0 & $3.770+11$ & $3.682+11$ \\
\hline 11 & 1210.6 & $3.707-8$ & 2.575 & 23300 & 7.0 & $4.240+11$ & $4.148+11$ \\
\hline 12 & 1210.4 & $5.010-8$ & 5.154 & 23180 & 7.0 & $4.370+11$ & $4.282+11$ \\
\hline 13 & 1210.0 & $7.665-8$ & $1.032+1$ & 23050 & 7.0 & $4.500+11$ & $4.406+11$ \\
\hline 14 & 1209.3 & $1.331-7$ & $2.065+1$ & 22830 & 7.0 & $4.760+11$ & $4.672+11$ \\
\hline 15 & 1208.0 & $2.455-7$ & $4.133+1$ & 22370 & 7.0 & $5.420+11$ & $5.323+11$ \\
\hline 16 & 1205.9 & $4.409-7$ & $8.271+1$ & 21140 & 7.0 & $5.950+11$ & $5.836+11$ \\
\hline 17 & 1204.1 & $6.346-7$ & $1.655+2$ & 17480 & 7.0 & $6.610+11$ & $6.463+11$ \\
\hline 18 & 1202.6 & $8.083-7$ & $3.313+2$ & 14580 & 7.0 & $7.250+11$ & $7.051+11$ \\
\hline 19 & 1200.9 & $1.025-6$ & $6.632+2$ & 13470 & 7.0 & $8.250+11$ & $7.889+11$ \\
\hline 20 & 1198.9 & $1.316-6$ & $1.327+3$ & 12050 & 7.0 & $9.400+11$ & $8.626+11$ \\
\hline 21 & 1197.5 & $1.598-6$ & $2.657+3$ & 10600 & 7.0 & $1.400+12$ & $1.129+12$ \\
\hline 22 & 1197.0 & $1.804-6$ & $5.317+3$ & 9390 & 7.0 & $3.500+12$ & $1.773+12$ \\
\hline 23 & 1196.7 & $2.071-6$ & $1.064+4$ & 8940 & 7.0 & $9.000+12$ & $2.468+12$ \\
\hline 24 & 1196.4 & $2.505-6$ & $2.130+4$ & 8780 & 6.5 & $1.050+13$ & $2.301+12$ \\
\hline 25 & 1196.0 & $3.307-6$ & $4.263+4$ & 8640 & 6.5 & $1.300+13$ & $2.305+12$ \\
\hline 26 & 1195.2 & $4.826-6$ & $8.533+4$ & 8430 & 6.0 & $1.000+13$ & $1.565+12$ \\
\hline 27 & 1193.1 & $7.715-6$ & $1.708+5$ & 8230 & 6.0 & $6.000+12$ & $8.895+11$ \\
\hline 28 & 1184.9 & $1.346-5$ & $3.418+5$ & 7970 & 6.0 & $2.460+12$ & $3.587+11$ \\
\hline 29 & 1160.4 & $2.455-5$ & $6.841+5$ & 7830 & 6.0 & $2.960+12$ & $3.443+11$ \\
\hline 30 & 1122.3 & 4.559-5 & $1.369+6$ & 7510 & 6.0 & $3.660+12$ & $2.703+11$ \\
\hline 31 & 1065.7 & $8.484-5$ & $2.741+6$ & 7100 & 6.0 & $4.620+12$ & $2.131+11$ \\
\hline 32 & 981.7 & $1.587-4$ & $5.485+6$ & 6600 & 5.0 & $5.910+12$ & $2.178+11$ \\
\hline 33 & 891.9 & $2.989-4$ & $1.098+7$ & 6300 & 5.0 & $1.270+13$ & $2.876+11$ \\
\hline 34 & 835.6 & $5.695-4$ & $2.197+7$ & 6150 & 5.0 & $4.500+13$ & $4.042+11$ \\
\hline 35 & 771.2 & $1.096-3$ & $4.398+7$ & 6000 & 4.5 & $5.280+13$ & $4.072+11$ \\
\hline 36 & 705.1 & $2.121-3$ & $8.802+7$ & 5800 & 4.4 & $1.330+14$ & $5.083+11$ \\
\hline 37 & 673.0 & 4.058-3 & $1.762+8$ & 5450 & 4.3 & $5.900+14$ & $8.094+11$ \\
\hline 38 & 644.1 & $7.899-3$ & $3.526+8$ & 5150 & 4.2 & $1.000+15$ & $1.019+12$ \\
\hline 39 & 608.6 & $1.533-2$ & $7.057+8$ & 4820 & 4.1 & $1.510+15$ & $1.306+12$ \\
\hline 40 & 561.9 & $3.073-2$ & $1.413+9$ & 4560 & 4.0 & $2.200+15$ & $1.780+12$ \\
\hline 41 & 500.0 & $5.778-2$ & $2.827+9$ & 4470 & 3.9 & $3.200+15$ & $2.285+12$ \\
\hline 42 & 427.4 & $1.136-1$ & $5.659+9$ & 4540 & 3.8 & $6.000+15$ & $2.484+12$ \\
\hline 43 & 353.2 & $2.255-1$ & $1.333+10$ & 4670 & 3.6 & $1.200+16$ & $2.723+12$ \\
\hline 44 & 280.5 & $4.492-1$ & $2.267+10$ & 4900 & 3.5 & $2.500+16$ & $3.475+12$ \\
\hline 45 & 206.3 & $9.028-1$ & $4.537+10$ & 5120 & 3.3 & $4.800+16$ & $5.218+12$ \\
\hline 46 & 125.3 & 1.854 & $9.080+10$ & 5900 & 3.1 & $9.200+16$ & $1.846+13$ \\
\hline 47 & 133 & 3.808 & $1.817+11$ & 7300 & 3.0 & $1.180+17$ & $2.238+14$ \\
\hline 48 & -152.7 & 7.298 & $3.638+11$ & 8600 & 3.0 & $1.355+17$ & $1.265+15$ \\
\hline
\end{tabular}




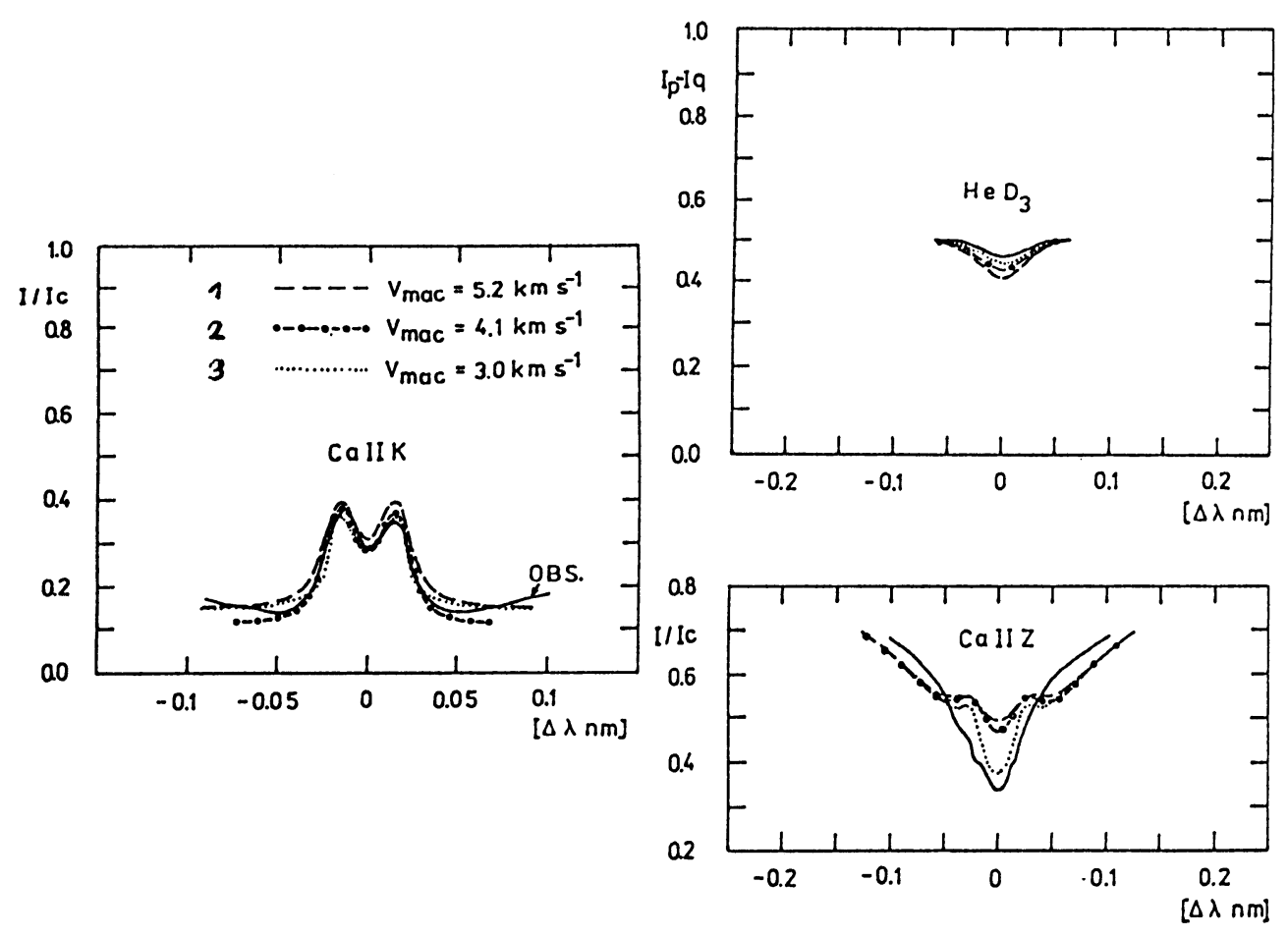

Fig. 2. Computed and observed intensity profiles of the plage for $\mathrm{Ca} \mathrm{II} \mathrm{K}, \mathrm{He} \mathrm{D}_{3}$ and infrared Ca II Z lines.

We can see that model 3 best fits the observational profiles. (The double reversal in the internal parts of the observational profile of the IR Ca II line is weak due to seeing over a long exposure time.) The principal parameters of the model 3 are listed in Table I. As shown in Figure 1, the model assumes a rapid enhancement of the hydrogen number density over a small range in height. From Table I we determine that at these heights the gas pressure $\left(P_{1}=N k T+\rho v^{2} / 2\right)$ exceeds the hydrostatic pressure, $\left(P_{2}=m g\right.$, where $g$ is gravitational acceleration).

To balance the plage atmosphere we assume the additional magnetic pressure. In the higher layers of the atmosphere (where the preasure is relative low) the magnetic field may play an important role as a source of the pressure and at the same time its geometrical configuration can limit the expansion of the solar plasma. It may enhance the number density at the crucial height. A magnetic field in the range 5-100 $\mathrm{G}$ is enough to supply the pressure needed to balance the atmosphere.

The possibility of significantly affecting the hydrogen number density by magnetic fields must be further examined. Certainly it would be useful to take into account all possible pressure components in the calculations of pressure balance in 
the solar atmosphere. To verify the above stated hypothesis, more precise observations are needed, which are currently under way.

\section{Acknowledgements}

This work has been supported under Grant GA 494/1991 by the Slovak Academy of Sciences.

\section{References}

Avret, E.H., Loeser, R.: 1969, Smithsonian Astrophys.Obs Spec.Rept. 303.

Ayres, T.R., Testerman, L. and Brault, J.W.: 1986, A strophys.J. 304, 542.

Baranovsky, E.A., Severny, A.B.: 1979, Izv.Krimsk.Astrofiz.Obs. 60, 99.

Baranovsky, E.A., Stepanian, N.N.: 1976, Izv.Krimsk.Astrofiz.Obs. 55, 14.

Baranovsky, E.A., Stepanian, N.N.: 1979, Izv.Krimsk.Astrofiz.Obs. 60, 135.

Basri, G.S., Linsky, J.L., Bartoe, J.D.F., Brueckner, G. and Van Hooser, M.E.: 1979, A strophys.J. $230,924$.

Jebsen, D.E., Mitchel, Jr., E.: 1978, Solar Phys. 57, 309.

Kelch, W.L., Linsky, J.L.: 1978, Solar Phys. 58, 37.

Kučera, A., Rybák, J., Minarovjech, M., Novocký, D., Saniga, M.: 1990, Astrophys. Space Sci. 17, 281.

Kučera, A., Scherbakova, Z., and Baranovsky, E.: 1990, in P. Ulmschneider, E.R. Priest, and R. Rosner (eds.), Mechanisms of Chromospheric and Coronal Heating, p. 109.

LaBonte, B.J.: 1986a, Astrophys J. Suppl. 62, 229.

LaBonte, B.J.: 1986b, Astrophys J. Suppl. 62, 241.

Lemaire, P., Goutterbroze, P., Vial, J.C. and Artzner, G.E.: 1981, Astron. Astrophys. $103,160$.

Shine, R.A., Linsky, J.L.: 1972, Solar Phys. 25, 357.

Shine, R.A., Linsky, J.L.: 1974, Solar Phys. 39, 49. 